\title{
A PET study examining pharmacokinetics and dopamine transporter occupancy of two long-acting formulations of methylphenidate in adults
}

\author{
THOMAS J. SPENCER ${ }^{1,2,4}$, ALI A. BONAB ${ }^{3,4,5}$, DARIN D. DOUGHERTY ${ }^{2,4}$, \\ JESSICA MARTIN $^{1}$, TARA MCDONNELL ${ }^{1}$ and ALAN J. FISCHMAN ${ }^{4,5}$ \\ ${ }^{1}$ Pediatric Psychopharmacology Unit, ${ }^{2}$ Psychiatry Service Massachusetts General Hospital, \\ Department of Psychiatry, Harvard Medical School, Boston; ${ }^{3}$ Department of Nuclear \\ Medicine, Massachusetts General Hospital, Harvard Medical School, Boston; \\ ${ }^{4}$ Harvard Medical School and ${ }^{5}$ Shriners Hospitals for Children, Boston, MA, USA
}

Received July 24, 2009; Accepted August 17, 2009

DOI: 10.3892/ijmm_00000339

\begin{abstract}
The delivery systems of two long-acting formulations of methylphenidate (MPH) were designed for different durations. Diffucaps bead-delivery system (DBDS)-MPH was designed to last $8 \mathrm{~h}$ and osmotically controlled-release oral delivery system (OROS)-MPH was designed to last $12 \mathrm{~h}$. While the plasma pharmacokinetics and timing of efficacy have been studied, the corresponding central nervous system dopamine transporter (DAT) occupancies are unknown. In this study, 21 healthy volunteers underwent PET imaging with ${ }^{11} \mathrm{C}$ Altropane before and after administration of oral doses of DBDS-MPH and OROS-MPH. Each subject received $40 \mathrm{mg}$ DBDS-MPH and $36 \mathrm{mg}$ OROS-MPH on different days. PET imaging occurred at $10 \mathrm{~h}$ after dosing. Each subject was injected with $5 \mathrm{mCi}$ of ${ }^{11} \mathrm{C}$ Altropane and serial images of the brain were acquired over 60 min with a Siemens $\mathrm{HR}^{+}$PET camera. Binding potentials $\left(\mathrm{BP}, \mathrm{k}_{3} / \mathrm{k}_{4}\right.$ ) were calculated from time-activity curves using the simplified reference region method with cerebellum as reference. Transporter occupancy was calculated by standard methods. At $10 \mathrm{~h}$, plasma d-MPH levels were lower (3.8 \pm 1.2 vs. $5.2 \pm 2.0)$ and brain DAT occupancy was lower $(34.8 \pm 12.9$ vs. $44.3 \pm 11.8)$ for DBDSMPH than OROS-MPH. Across the range of values, for each unit of change in plasma d-MPH level there was a larger
\end{abstract}

Correspondence to: Dr Thomas Spencer, Pediatric Psychopharmacology Unit (ACC-725), Massachusetts General Hospital, 55 Fruit Street, Boston, MA 02114, USA

E-mail: tspencer@partners.org

Abbreviations: DAT, dopamine transporter; MPH, methylphenidate; ADHD, attention deficit hyperactivity disorder, PET, positron emission tomography; FWHM, full width at half maximum; TAC, time-activity curve

Key words: dopamine transporter, methylphenidate, attention deficit hyperactivity disorder, positron emission tomography, controlledrelease, binding potential change in DAT occupancy with the DBDS-MPH formulation than with the OROS-MPH formulation. As predicted from previous pharmacokinetic and efficacy data, the average plasma level and DAT occupancy of $36 \mathrm{mg}$ OROS-MPH was $>40 \mathrm{mg}$ DBDS-MPH at $10 \mathrm{~h}$. Moreover, a relatively small difference in plasma levels $(1.4 \mathrm{ng} / \mathrm{ml}$ at $10 \mathrm{~h})$ was associated with a more impressive difference in DAT occupancy ( 10\% at $10 \mathrm{~h})$.

\section{Introduction}

The stimulant methylphenidate (MPH) remains a mainstay of treatment for attention deficit hyperactivity disorder (ADHD) with over a hundred studies documenting its safety and efficacy in pediatric and adult ADHD (1). Because of the short half life of MPH, a new generation of long acting MPH formulations has emerged that has greatly improved the management of patients with ADHD across the lifecycle. While the pharmacokinetic (PK) data from studies using these formulations support their longer duration of action, whether peripheral plasma kinetics match their effects in the brain remain unknown.

Since the main target of MPH in the brain is the striatal dopamine transporter (DAT), (2) measuring central DAT binding can elucidate the central kinetic effects of MPH formulations. A highly sensitive methodology has been developed to measure drug occupancy of the DAT in the brain using the radioligand ${ }^{11} \mathrm{C}$ Altropane to label the DAT and positron emission tomography (PET) for detection (3). Because of the sensitivity of this methodology, low radiation exposure permits repeated imaging, allowing for the examination of the kinetics of CNS DAT receptor occupancy in the living human brain. Similar PET methodology has been previously used to document the CNS pharmacokinetics of other psychiatric drugs (4-8).

Using this methodology, we recently contrasted the central effects of immediate release (IR)-MPH and the long-acting osmotically controlled-release oral delivery system (OROS)MPH in 12 healthy adults. (9) Subjects were randomized to 
receive single doses of IR-MPH or OROS-MPH using doses predicted to produce equivalent Cmax values (40 mg IR-MPH, $90 \mathrm{mg}$ OROS-MPH). Plasma d-MPH levels were obtained hourly for $10 \mathrm{~h}$, twice for each subject. DAT occupancies were measured at $1,3,5$ and $7 \mathrm{~h}$ using ${ }^{11} \mathrm{C}$ Altropane and PET. Results showed that despite similar Cmax for both formulations, treatment with OROS-MPH was associated with a greater Tmax, a later time to maximum CNS DAT occupancy consistent with the effects of a long-acting formulation.

Of the available second generation long-acting MPH formulations, OROS-MPH and Diffucaps bead-delivery system (DBDS)-MPH were specifically developed to produce an ascending PK profile aimed at enhancing efficacy by offsetting acute tolerance (10). However, these 'ascending' pharmacokinetics profiles are achieved through different delivery technologies. OROS-MPH was designed to deliver $22 \%$ of its MPH immediately through its overcoat of MPH on the surface of the tablet and the rest slowly throughout the day through its delayed release mechanism relying on an expanding push compartment that releases a pre-specified amount of MPH while transitioning through the gut across the day. In contrast, the ascending pharmacokinetics profile of DBDSMPH is achieved through the use of $30 \%$ immediate release and $70 \%$ delayed release beads. At comparable doses, DBDSMPH is associated with greater plasma concentrations at early times $(1.5,3,4 \mathrm{~h})$ whereas OROS-MPH is associated with greater plasma concentrations $(8,10,12 \mathrm{~h})$ at later times (11).

These two formulations also differ in their duration of effects. While OROS-MPH was designed to replace three doses of IR-MPH given $4 \mathrm{~h}$ apart with an expected duration of effect of $12 \mathrm{~h}$, DBDS-MPH was designed to replace two doses of IR-MPH with an expected duration of effect of $8 \mathrm{~h}$. Whether the similarities (ascending pattern) and differences (duration of action) of these two delivery systems will lead to parallel effects on DAT occupancy in the brain remain unknown.

Evaluating whether different long-acting formulations of MPH differ in their central effects has important implications. Linking plasma kinetics with brain activity of MPH could further our understanding of the underlying central effects of MPH in humans. Such information can help clarify whether the molecular physiology of central MPH action (DAT brain occupancy) parallels its peripheral pharmacokinetic properties (plasma level). Whether the clinical effects of MPH correlate with its central or peripheral kinetics can be of great value to clinicians and to the public in selecting the most appropriate MPH treatment for ADHD in clinical practice. This type of research can provide novel and unique information towards a better understanding of the mechanisms of action of longacting stimulant formulations. Considering the high prevalence of ADHD in children and adults and its high associated morbidity and disability, a better understanding of the time course of brain actions of different MPH formulations will facilitate access to a highly effective treatment for millions of afflicted individuals.

The main aim of this study was to investigate the molecular physiology of the brain action of OROS-MPH and DBDS$\mathrm{MPH}$ using PET scanning with ${ }^{11} \mathrm{C}$ Altropane as the ligand. Since these formulations are largely differentiable in their

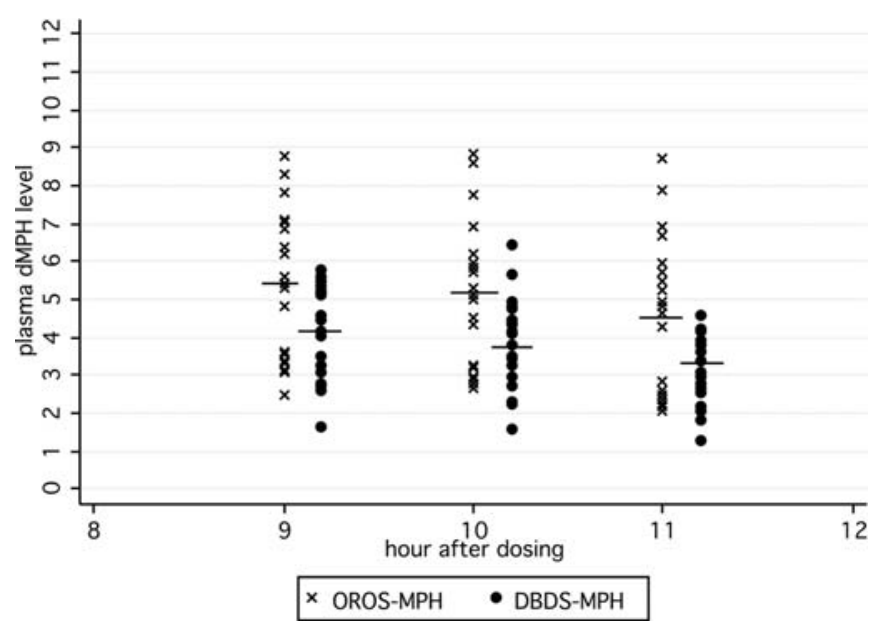

Figure 1. Plasma pharmacokinetics of d-Methylphenidate (DBDS-MPH values offset to aid visualization).

duration of action, our study focused on their delayed release phase. Based on their pharmacokinetic designs, we hypothesized that at similar doses, OROS-MPH will have to higher plasma d-MPH levels and greater CNS DAT occupancy $10 \mathrm{~h}$ after administration than those of DBDS-MPH.

\section{Materials and methods}

Twenty-one healthy human subjects between 18 and 55 years were sequentially recruited from advertisements. All subjects were right-handed and in good health. All subjects had a complete medical and psychiatric history and physical examination before imaging. None had any DSM-IV axis I disorders including ADHD as well as current or past drug or alcohol abuse. In addition, none had a history of exposure to psychotropic medicines (including stimulants) or tobacco. In females, inquiry about the subject's current reproductive status was also made. Furthermore, all subjects had an ECG, full blood count, blood chemistries, urinalysis (including drug screen and, in females, a pregnancy test). All studies were approved by the Subcommittee on Human Studies of the Massachusetts General Hospital.

Procedures. Subjects underwent PET imaging before and after administration of oral doses of DBDS-MPH and OROS-MPH. Assignment of medication order was randomized by the MGH pharmacy. Subjects were blind to the order of medication assignment. Each subject received $40 \mathrm{mg}$ DBDS-MPH and $36 \mathrm{mg}$ OROS-MPH on different days: day 1: baseline scanning (one scan), day 2: scanning at $10 \mathrm{~h}$ after administration of medication (DBDS-MPH or OROS-MPH) and day 3: scanning at $10 \mathrm{~h}$ after administration of medication (DBDS-MPH or OROS-MPH). On days 2 and 3 of scanning, venous blood was drawn for quantification of plasma concentration of d-MPH at 9,10 and $11 \mathrm{~h}$ after medication administration.

PET imaging. Images were acquired using an $\mathrm{HR}^{+}$(CTI, Knoxville, TN) PET camera. The primary imaging parameters of the $\mathrm{HR}^{+}$camera are in-plane and axial resolution of 4.5-mm FWHM, 63 contiguous slices of 2.5-mm separation. 


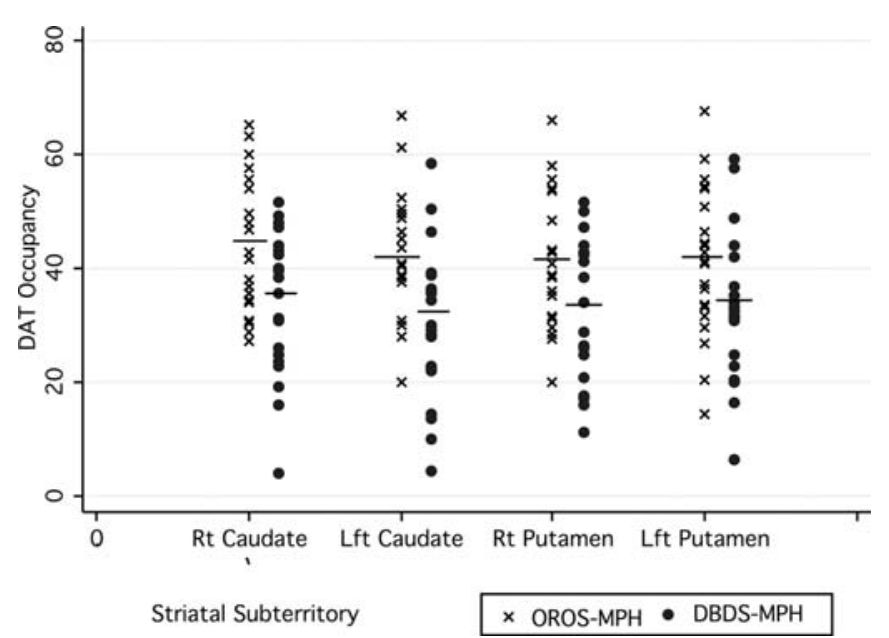

Figure 2. DAT Occupancy at $10 \mathrm{~h}$ by drug formulation.

Images were acquired in 3D mode and reconstructed using a filtered back projection algorithm to an in-plane resolution of 4.5-mm FWHM. Photon attenuation measurements were made with rotating pin sources containing ${ }^{68} \mathrm{Ge}$.

For each scan, $\sim 5 \mathrm{mCi}$ of ${ }^{11} \mathrm{C}$ Altropane was injected intravenously over $30 \mathrm{sec}$ and serial PET images acquired. Dynamic image collection started at the same time as the infusion and images were acquired in $15 \mathrm{sec}$ frames for the first $2.0 \mathrm{~min}$; in $1 \mathrm{~min}$ frames for the next $4.0 \mathrm{~min}$ and in $2 \mathrm{~min}$ frames for the last 27 frames; $60 \mathrm{~min}$ in all.

All projection data were corrected for non-uniformity of detector response, dead time, random coincidences, and scattered radiation. Regions of interest (ROIs) representing the striatum (left, right caudate nucleus and left, right putamen) and cerebellum were drawn manually on the PET images. This procedure was repeated for all slices in which the structures were visualized at full intensity (away from edge slices). For each frame, ROIs of like structures were averaged to yield average striatal and cerebellar time activity curves (TACs). In previous studies, we determined that the reference (cerebellum) TACs were not affected by the DAT inhibitor (MPH).

Statistical analysis. Categorical data were analyzed by Chi-square analysis, continuous parametric data by an unpaired t-test and the rank sum test for nonparametric data. Associations between continuous variables were evaluated using Pearson correlations. We chose a significance level of 0.05 . All tests were two-tailed.

\section{Results}

Pharmacokinetic profile of DBDS and OROS MPH. To relate plasma levels of d-MPH to DAT occupancy, plasma d-MPH was measured at 9,10 and $11 \mathrm{~h}$ after receiving $36 \mathrm{mg}$ of OROS-MPH or $40 \mathrm{mg}$ of DBDS-MPH (Fig. 1). As expected, plasma d-MPH levels were lower for DBDS-MPH than OROS-MPH at $9 \mathrm{~h}[4.1 \pm 1.3$ vs. $5.4 \pm 2.0, \mathrm{t}=4.3, \mathrm{df}(18)$, $\mathrm{p}<0.001] ; 10$ h [3.8 \pm 1.2 vs. $5.2 \pm 2.0, \mathrm{t}=5.2, \mathrm{df}(20), \mathrm{p}<0.0001]$; and $11 \mathrm{~h}[3.2 \pm 0.9$ vs. $4.3 \pm 1.8, \mathrm{t}=4.3, \mathrm{df}(17), \mathrm{p}<0.001]$ (Fig. 1 ).
A

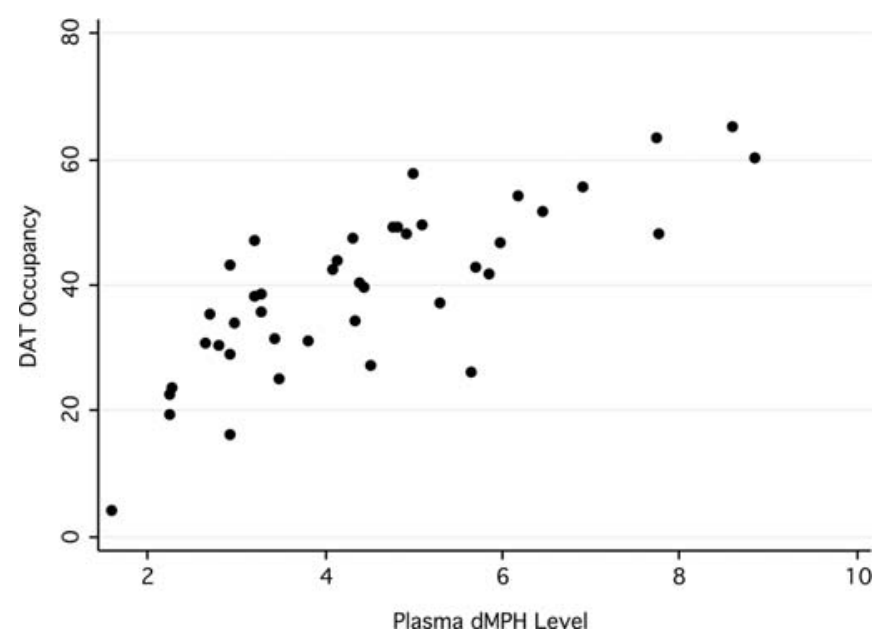

B

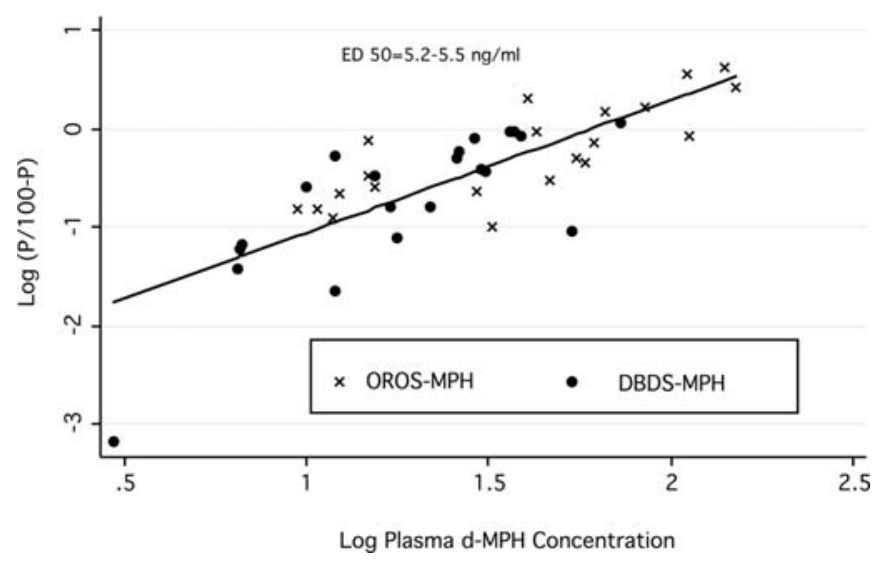

C

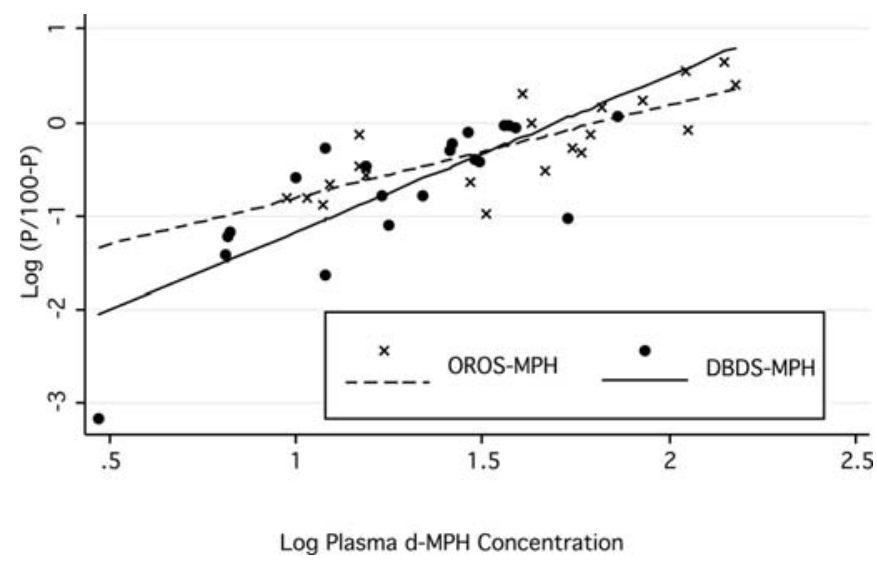

Figure 3. Plasma d-MPH level and DAT occupancy: raw data (A). Plasma dMPH level and DAT occupancy: log transformed data, overall slope (B). Plasma dMPH level and DAT occupancy: log transformed data, individual slopes (C).

DAT occupancy in the striatum. d-MPH occupancy of brain DAT was measured at $10 \mathrm{~h}$ after administration of $36 \mathrm{mg}$ of OROS-MPH or $40 \mathrm{mg}$ of DBDS-MPH. Fig. 2 shows that at $10 \mathrm{~h}$, DAT occupancy of OROS-MPH was significantly greater than DBDS-MPH in all sub-territories of the striatum: right caudate $(44.3 \pm 11.8$ vs. $34.8 \pm 12.9, \mathrm{t}=3.9, \mathrm{df}=20, \mathrm{p}=0.001)$, left 
caudate $(42.7 \pm 10.9$ vs. $30.9 \pm 13.8, \mathrm{t}=5.1, \mathrm{df}=20, \mathrm{p}=0.001)$, right putamen $(41.6 \pm 11.7$ vs. $32.1 \pm 12.3, \mathrm{t}=3.8, \mathrm{df}=20, \mathrm{p}=0.001)$ and left putamen $(41.3 \pm 13.1$ vs. $33.0 \pm 12.8, \mathrm{t}=3.4, \mathrm{df}=20$, $\mathrm{p}=0.003$ ).

DAT occupancy was significantly correlated with plasma concentration of d-MPH (correlation coefficient 0.78, $\mathrm{t}=8.0$, $\mathrm{p}<0.000$ ) (Fig. 3A). Using log transformation of DAT data, similar to the analysis of DAT findings by Volkow et al (2), a plasma concentration of 5.2-5.5 ng/ml (depending on subterritory) of d-MPH was associated with a $50 \%$ blockade of DAT (Fig. 3B). Furthermore, the slope of the relationship of plasma d-MPH concentration to DAT occupancy was significantly greater for the DBDS-MPH formulation than that of OROS-MPH and the plasma MPH concentration by drug formulation interaction was significant $(\mathrm{t}=9.5, \mathrm{df}=40$, $\mathrm{p}<0.0001$ ) (Fig. 3C).

\section{Discussion}

This study compared the relationship between peripheral pharmacokinetics and central DAT occupancies of two longacting oral delivery system formulations of MPH. DBDSMPH was designed to release d-MPH in escalating amounts over $8 \mathrm{~h}$, whereas OROS-MPH was designed to release dMPH over 12 h. Despite similar dosing, OROS-MPH had greater plasma concentrations and greater brain effects (occupancy of the DAT) at $10 \mathrm{~h}$ compared with DBDS-MPH. These results support the hypotheses that peripheral and brain pharmacokinetic profiles can be accurately predicted based on the delivery system profile of the formulation.

The difference in plasma d-MPH concentrations at 9, 10 and $11 \mathrm{~h}$ of $40 \mathrm{mg}$ DBDS-MPH and $36 \mathrm{mg}$ of OROS-MPH are consistent with previous PK studies for both formulations (11). Gonzalez et al documented consistent differences in plasma d-MPH levels for several comparable doses of DBDSMPH and OROS-MPH (11). While these investigators determined that total exposure $\left(\mathrm{AUC}_{0-24}\right)$ was comparable between the two formulations at the doses studied, earlier exposure was greater for DBDS-MPH $\left(\mathrm{AUC}_{0-6}\right.$, mean plasma d-MPH at $1.5,3,4 \mathrm{~h}$ ) and later exposure was greater for OROS-MPH (mean plasma d-MPH at 8,10,12 h). The authors concluded that while both long-acting formulations provided a similar total exposure; they were not bioequivalent.

While our pharmacokinetic results are consistent with previous pharmacokinetic studies, it is striking that a relatively small difference in plasma levels $(1.4 \mathrm{ng} / \mathrm{ml}$ at $10 \mathrm{~h})$ was associated with a more impressive difference in DAT occupancy $(\sim 10 \%$ at $10 \mathrm{~h})$. A pharmacodynamic study by Swanson et al underscores the clinical significance of such a difference in DAT occupancy. The study of Swanson et al compared the effects of comparable doses of DBDS-MPH and OROS-MPH in children with ADHD in an analogue classroom model (12). These investigators showed that DBDSMPH was associated with greater efficacy at early hours (1.5, $3,4.5 \mathrm{~h}$ ), whereas OROS-MPH was associated with greater efficacy at later hours (12 h) on all measures assessed. Better efficacy at any point in time was predicted by higher plasma d-MPH concentrations.

While plasma concentrations predicted DAT occupancy with both formulations of MPH, there was a stronger correlation between plasma d-MPH levels and DAT occupancy with the DBDS-MPH formulation than with the OROS-MPH formulation. This finding is similar to our previous finding that there was a stronger correlation between plasma d-MPH levels and DAT occupancy with the IR-MPH formulation than with the OROS-MPH formulation (9). Although the reasons for this finding are unclear, in both comparisons there was a higher correlation in the formulation associated with more rapid change of plasma levels. In other words, when the delivery system is more rapid, for each unit of change in plasma MPH level there is a larger change in DAT occupancy. It is possible that plasma kinetics of OROS-MPH and DBDSMPH produce different physiologic outcomes due to rates of transport across the blood-brain barrier and the effects of CNS kinetics with DAT binding itself. More work is needed to confirm this finding and elucidate its meaning.

Our results should be viewed in light of some methodological limitations. Since we did not determine the phase of the menstrual cycle, this may be a source of variability in DAT occupancy (13). Since we did not do Magnetic resonance imaging (MRI ) scans, we could not rule out brain pathology. However, brain pathology was unlikely in normal subjects. While ROIs can be drawn with MRI scans, PET scans with altropane clearly outline the salient structures (striatum, cerebellum). ROIs were chosen to be well inside the boundaries to avoid partial volume effects. The same ROIs are used for each subsequent scan on each individual subject. Moreover, in a previous study (9) our IR-MPH DAT occupancies matched those of Volkow et al (2).

Despite these considerations, our results provide further documentation that peripheral and brain pharmacokinetic profiles of long-acting formulations of MPH can be accurately predicted based on the delivery system profile of the formulation used. Plasma levels of d-MPH were greater with OROS-MPH than DBDS-MPH from 9 to $11 \mathrm{~h}$ and DAT occupancy of OROS-MPH was greater than DBDS-MPH at $10 \mathrm{~h}$. Higher plasma levels were associated with higher occupancy of the DAT. These results advance our understanding of the underlying central effects of MPH in humans and identify important differences in the pharmacokinetic and pharmacodynamic effects of long-acting formulations.

\section{Acknowledgements}

This study was supported by funding from McNeil Pharmaceuticals. Dr Thomas Spencer receives research support from, is a speaker for or is on the Advisory Board of the following sources: Shire Laboratories, Inc., Eli Lilly \& Company, GlaxoSmith Kline, Janssen Pharmaceutical, McNeil Pharmaceutical, Novartis Pharmaceuticals, Cephalon, Pfizer and the National Institute of Mental Health.

\section{References}

1. A 14-month randomized clinical trial of treatment strategies for attention-deficit/hyperactivity disorder. The MTA Cooperative Group. Multimodal Treatment Study of Children with ADHD. Arch Gen Psychiatry 56: 1073-1086, 1999.

2. Volkow ND, Wang GJ, Fowler JS, Gatley SJ, Logan J, Ding YS, Hitzemann R and Pappas N: Dopamine transporter occupancies in the human brain induced by theraputic doses of oral methylphenidate. Am J Psychiatry 155: 1325-1331, 1988. 
3. Fischman AJ, Bonab AA, Babich JW, Livni E, Alpert NM, Meltzer PC and Madras BK: $\left[{ }^{11} \mathrm{C},{ }^{127} \mathrm{I}\right]$ Altropane: a highly selective ligand for PET imaging of dopamine transporter sites. Synapse 39: 332-342, 2001.

4. Fischman AJ, Bonab AA, Babich JW, Alpert NM, Rauch SL, Elmaleh DR, Shoup TM, Williams SA and Rubin RH: Positron emission tomographic analysis of central 5-hydroxytryptamine-2 receptor occupancy in healthy volunteers treated with the novel antipsychotic agent, ziprasidone. J Pharmacol Exp Ther 279: 939-947, 1996

5. Fischman AJ, Alpert NM and Rubin RH: Pharmacokinetic imaging: a noninvasive method for determining drug distribution and action. Clin Pharmacokinet 41: 581-602, 2002.

6. Fischman AJ, Alpert NM, Babich JW and Rubin RH: The role of positron emission tomography in pharmacokinetic analysis. Drug Metab Rev 29: 923-956, 1997.

7. Salazar DE and Fischman AJ: Central nervous system pharmacokinetics of psychiatric drugs. J Clin Pharmacol (Suppl): S10-S12, 1999.

8. Christian BT, Livni E, Babich JW, Alpert NM, Dischino DD, Ruediger E, Salazar DE, Ford NF and Fischman AJ: Evaluation of cerebral pharmacokinetics of the novel antidepressant drug, BMS-181101, by positron emission tomography. J Pharmacol Exp Ther 279: 325-331,1996.
9. Spencer TJ, Biederman J, Ciccone PE, Madras BK, Dougherty DD, Bonab AA, Livni E, Parasrampuria DA and Fischman AJ: PET study examining pharmacokinetics, detection and likeability, and dopamine transporter receptor occupancy of short- and long-acting oral methylphenidate. Am J Psychiatry 163: 387-395, 2006.

10. Swanson J, Gupta S, Lam A, Shoulson I, Lerner M, Modi N, Lindemulder E and Wigal S: Development of a new once-aday formulation of methylphenidate for the treatment of ADHD: Proof of concept and proof of product studies. Arch Gen Psychiatry 60: 204-211, 2003.

11. Gonzalez MA, Pentikis HS and Andrl N: Methylphenidate bioavailability from two extended-release formulations. Int $\mathrm{J}$ Clin Pharmacol Ther 40: 175-184, 2002.

12. Swanson JM, Wigal SB, Wigal T, Sonuga-Barke E, Greenhill LL, Biederman J, Kollins S, Nguyen AS, DeCory HH, Hirshe Dirksen SJ and Hatch SJ: A comparison of once-daily extended-release methylphenidate formulations in children with ADHD in the laboratory school (The Comacs study). Pediatrics 113: E206-E216, 2004.

13. Lammers CH, D'Souza U, Qin ZH, Lee SH, Yajima S and Mouradian MM: Regulation of striatal dopamine receptors by estrogen. Synapse 34: 222-227, 1999. 